\title{
Design of broadband filters and antennas for SAMBA
}

\section{Alexey Goldin, James J. Bock, Cynthia L. Hunt, Andrew E. Lange, Henry G. LeDuc, et al.}

Alexey Goldin, James J. Bock, Cynthia L. Hunt, Andrew E. Lange, Henry G. LeDuc, Anastasios Vayonakis, Jonas Zmuidzinas, "Design of broadband filters and antennas for SAMBA," Proc. SPIE 4855, Millimeter and Submillimeter Detectors for Astronomy, (17 February 2003); doi: 10.1117/12.459677

SPIE Event: Astronomical Telescopes and Instrumentation, 2002, Waikoloa, Hawai'i, United States 


\title{
Design of broadband filters and antennas for SAMBA
}

\author{
Alexey Goldin ${ }^{a}$, James J. Bock ${ }^{a}$, Cynthia Hunt ${ }^{b}$, \\ Andrew E. Lange ${ }^{b}$, Henry LeDuc ${ }^{a}$, Anastasios Vayonakis ${ }^{b}$, \\ Jonas Zmuidzinas ${ }^{b}$ \\ a JPL, 4800 Oak Grove drive, Pasadena, CA, USA \\ ${ }^{b}$ California Institute of Technology, Pasadena, CA,USA
}

July 30,2002

\begin{abstract}
We present a design for multipixel, multiband submillimeter instrument: SAMBA (Superconducting Antennacoupled, Multi-frequency, Bolometric Array). SAMBA uses antenna coupled bolometers and microstrip filters. The concept allows for a much more compact, multiband imager compared to a comparable feedhorn-coupled bolometric system. SAMBA incorporates an array of slot antennas, superconducting transmission lines, a wide band multiplexer and superconducting transition edge bolometers. The transition-edge film measures the millimeter-wave power deposited in the resistor that terminates the transmission line.
\end{abstract}

\section{INTRODUCTION}

SAMBA, Superconducting Antenna-coupled, Multi-frequency, Bolometric Array architecture promises several advantages compared to existing systems. Unlike phase sensitive detection techniques, direct detectors can be photon-noise limited under extremely low-background conditions, even those of dispersive spectroscopy with a cooled aperture. Antenna-coupled bolometers provide greatly reduced thermally active area, volume, heat capacity and thermal conductivity, and improved rejection of stray light and out-of-band radiation. The large sub-K feedhorn optics and metal-mesh filters currently required by missions such as Planck and FIRST which comprise $>95 \%$ of the sub-K focal plane mass and volume would be eliminated by the new focal plane architectures.

To realize these advantages, we need to refine and combine the following existing technologies: wideband superconducting stripline filters, long superconducting transmission lines, large area synthesized phased arrays using slot antennas.

\section{FILTERS}

Spectral filtering is done by microstrip filters, which have low loss and excellent out-of-band rejection. They replace the much larger and more massive metal-mesh filters required by bolometers with radiation absorbers. The high frequency blocking requirements of mm-wave bolometers are quite severe. However the blocking requirements for antenna-coupled bolometers are relatively modest since superconducting microstrips do not efficiently propagate high frequency radiation at frequencies above superconductivity gap energy limit.

Using prototype from Blinchikoff and Zverev, ${ }^{1}$ we designed a lumped element prototype. This Chebyshev filter provides $30 \%$ bandpass with three poles. (figure 2). The capacitors and $L C$ elements were approximated by radial stubs and a combination of coplanar waveguide with microstrip. The filter was optimized with Supermix $^{2}$ - a C ++ library, developed in Caltech primarily for simulation of SIS mixers. To finalize the design, the components were analyzed using Sonnet package.

Send correspondence to Alexey Goldin:

E-mail: Alexey.Goldin@jpl.nasa.gov, Telephone: +1 (818) 393-4275, Address: 4800 Oak Grove Drive

M/S: $169-327$

Pasadena, CA 91109-8099 
Figure 1. Filter schematics. The center frequency is $100 \mathrm{GHz}$ and the transmission line impedance $-5 \Omega$. On the right: $350 \mathrm{GHz}$ band filter manufactured at Micro Devices Laboratory (MDL) at JPL.
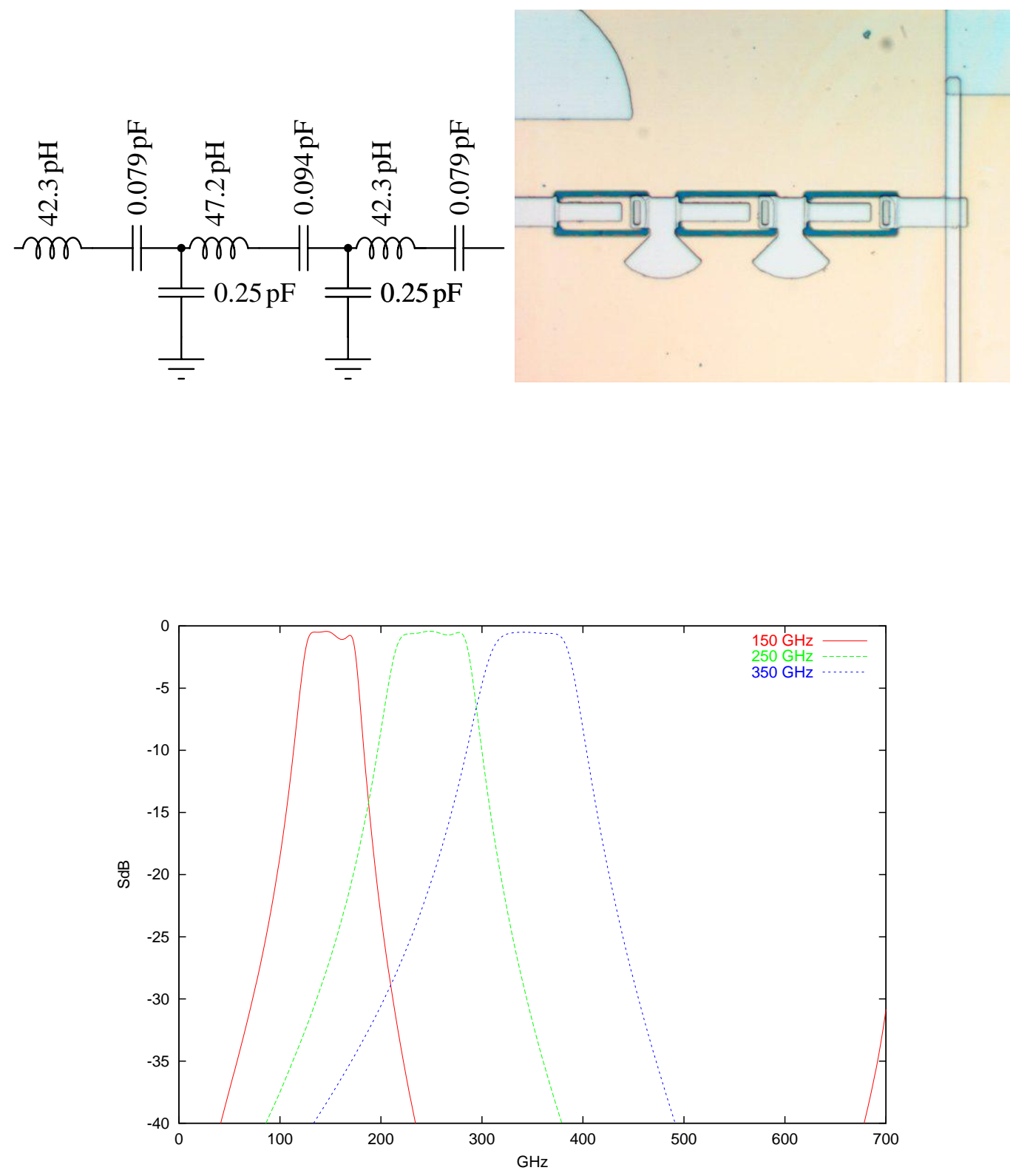

Figure 2. Transmission of three filters with bandpasses centered at 150, 250 and 350 GHz. Because of the small (compared to wavelength) size of the filter elements, the next resonance of the $150 \mathrm{GHz}$ filter does not start until approximately $700 \mathrm{GHz}$, well beyond the range of highest frequency filter, where it can be easily suppressed by a lowpass filter if necessary. 


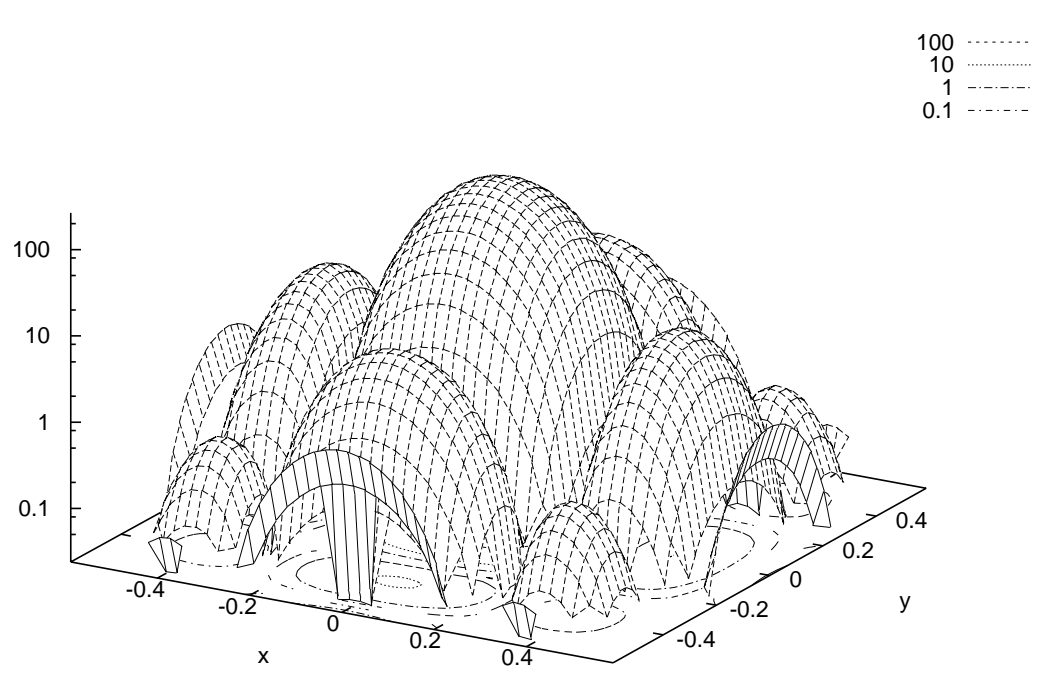

Figure 3. Beamshape of $100 \mathrm{GHz}$ array antenna with 15 by 16 slots vs. $\sin (\Theta)$. The array period is 700 microns, the slot length is 550 micron, the offset is 228 micron. The nearest sidelobes are at level of about 2.5 percent of the central peak.

\section{FOCAL PLANE ANTENNA}

The SAMBA architecture is uses a passive phased array antenna. A familiar analog is a phased array of transmitting antennas. The amplitude and phase of the electric field at each antenna control the pointing and far-field beam pattern of such a phased array. Rather than actively controlling the electric field of each antenna, SAMBA operates by passively adding the electric fields of each antenna in a phase-coherent manner to synthesize diffraction-limited beams at multiple frequencies. This architecture eliminates any need for coupling optics, such as feedhorns and lenses. It must be used in conjuction with a cold aperture stop to reduce sidelobe response.

The fundamental element of the array under development is planar array of slot antennas photolitographed on silicon. Many such antennas tuned to different frequencies can be located on one silicon wafer. The total size of every array is dictated by the size of Airy pattern at lowest frequency.

Depending on the application, the focal plane can be built in two different configurations: a single frequency, dual polarization array or single polarization, multiple frequency array. The dual polarization array provides simulateneous detection of both polarizations, a systems advantage for polarimetry. This architecture is simple to realize and does not require microstrip cross-overs. It can be modified to extract the $\mathrm{Q}$ and U STokes parameters simultaneously. The multifrequency focal plane uses focal plane area more efficiently. It requires cross-overs microstrip transmission line if we preserve the Airy diffraction limit at each wavelength.

\subsection{Dual polarization antenna}

The period $p$ of the array is limited strongly by the requirement $p<\lambda_{\min }$, where $\lambda_{\min }$ is the shortest wavelength of interest in silicon (to avoid scattering radiation into sidelobes and surface waves). Because of this restriction, we chose to operate at first resonance of the antenna rather the second in order to realize lower impedance and a wide band, as described in paper on slot antennas by Zmuidzinas et al.. ${ }^{3}$ The length of the single slot antenna designed for operation at in the second second resonance would exceed period of the array. 


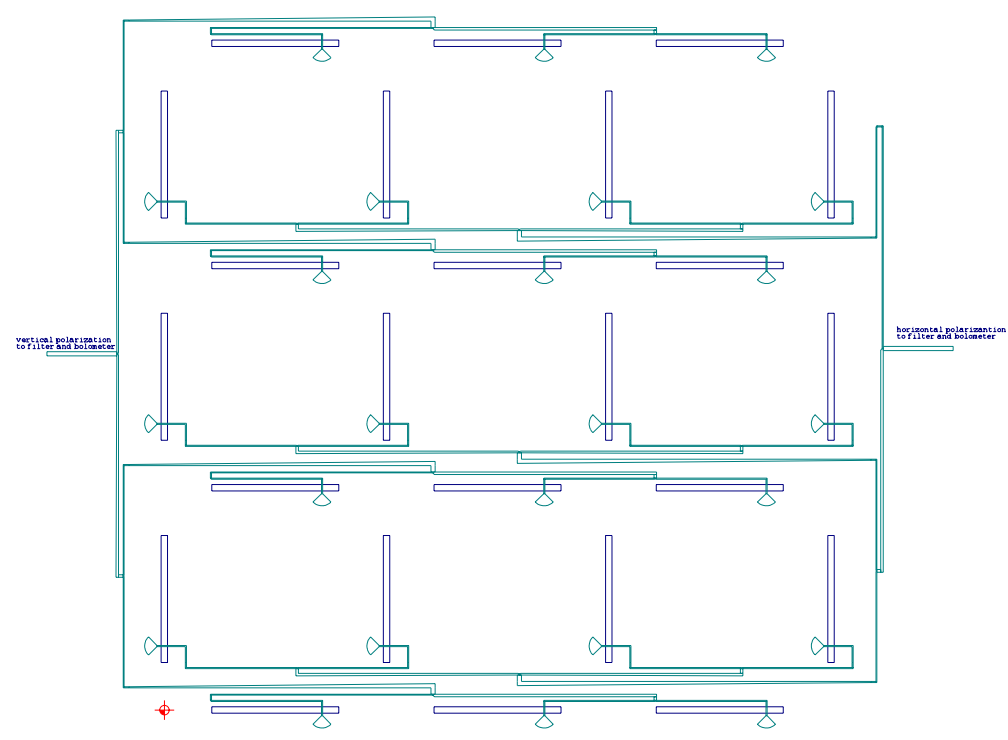

Figure 4. A small "baby" 4 by 3 dual polarizations array, shown for illustration purposes only. Dimensions are not to scale to avoid putting extremely fine details in a plot. The analyzed array has 15 by 16 antennas for each polarization. Stub capacitors are used to tune the inductive antenna to the microstrip feeds. We anticipate that the separate vertical and horizontal slots can be combined into cross slots with two feeds per cross.

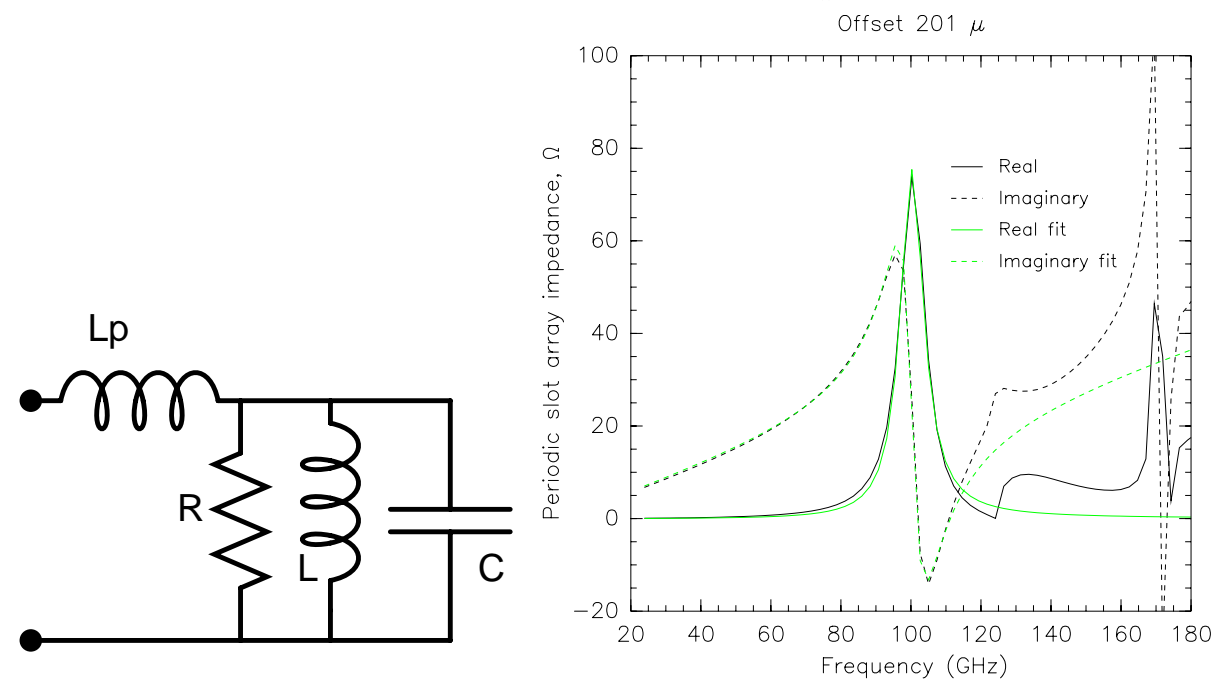

Figure 5. Equivalent circuit. Resistance $R$ simulates radiation into dielectric and air. This circuits fits antenna impedance to a few percent in the region of interest.

To better match antenna and microstrip impedance, we decided to place the microstrip feed off the center of each slot. This placement reduces antenna impedance and resonance quality factor $Q$ at a cost of introducing some reactive impedance. The reactive impedance can be tuned out by series capacitor.

We have done extensive EM simulations of this antenna array architecture using our own software, which generalizes method described in Eleftheriades and Rebeiz. ${ }^{4}$ The program is written in Numerical Python and scales well up to arrays of 16 by 16 antennas on $450 \mathrm{MHz}$ Pentium III desktop computer. We also wrote another 

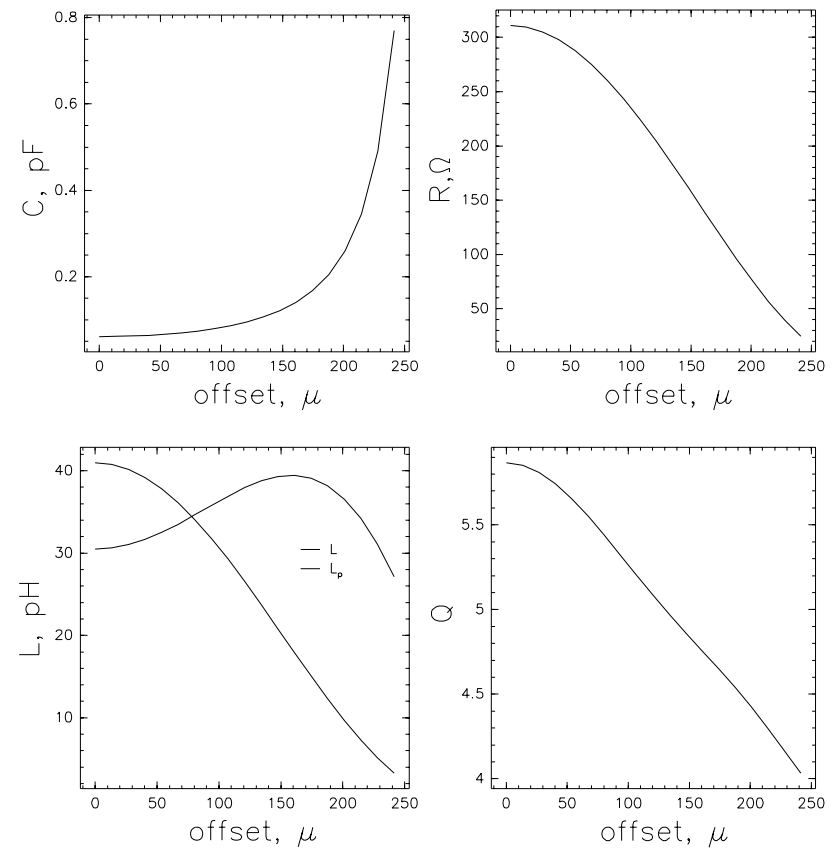

Figure 6. Equivalent circuit parameters as function of feed offset from the center.

program to simulate an infinite periodic array of slots. The output of these programs is antenna impedance and voltage distribution along the slot, which can be used to calculate the beammap. Both of these programs give very similar results for impedance of the antenna and beamshape, showing that the simpler infinite case is a good enough approximation.

The focal plane with a small number of pixels can be built on a single wafer having many antenna arrays tuned for different frequencies. The described design can be scaled up to 300-350 GHz. At higher frequencies densely packed transmission lines may require finer lithography, using JPL's e-beam system.

\subsection{Multi-frequency array}

Another possible architecture is single polarization, multi-frequency array.

The Figure 7 shows three arrays, designed to operated at bands $100 \mathrm{GHz}, 200 \mathrm{GHz}$ and $400 \mathrm{GHz}$ with a bandwidth of $30 \%$ each. The distance between taps (and between slots) is $174 \mu \mathrm{m}$, dictated by a highest frequency of operation. The beamsize of a single element of array is the same for every band, giving a total of $16400 \mathrm{GHz}$ pixels, $4200 \mathrm{GHz}$ pixels and $1100 \mathrm{GHz}$ pixel. The total size of the unit cell array is 64 by 64 taps, 11 by 11 millimeters. The maximum distance traveled by radiation is about 20 millimeters for the $100 \mathrm{GHz}$ detector. The impedance of such an array is real over a fairly broad region, which allows good coupling between antenna, filter bank spanning a wide frequency range, and detectors following the filter bank. This architecture is demonstrated in figures 7 and 8

The major sources of inefficiency are losses in transmission lines (see Vayonakis ${ }^{5}$ ), dielectric losses (unwanted radiation into lower hemisphere and reflection at dielectric interfaces) and losses due to impedance mismatch between antenna and transmission line. To reduce dielectric interface reflections, layered structure with graded 


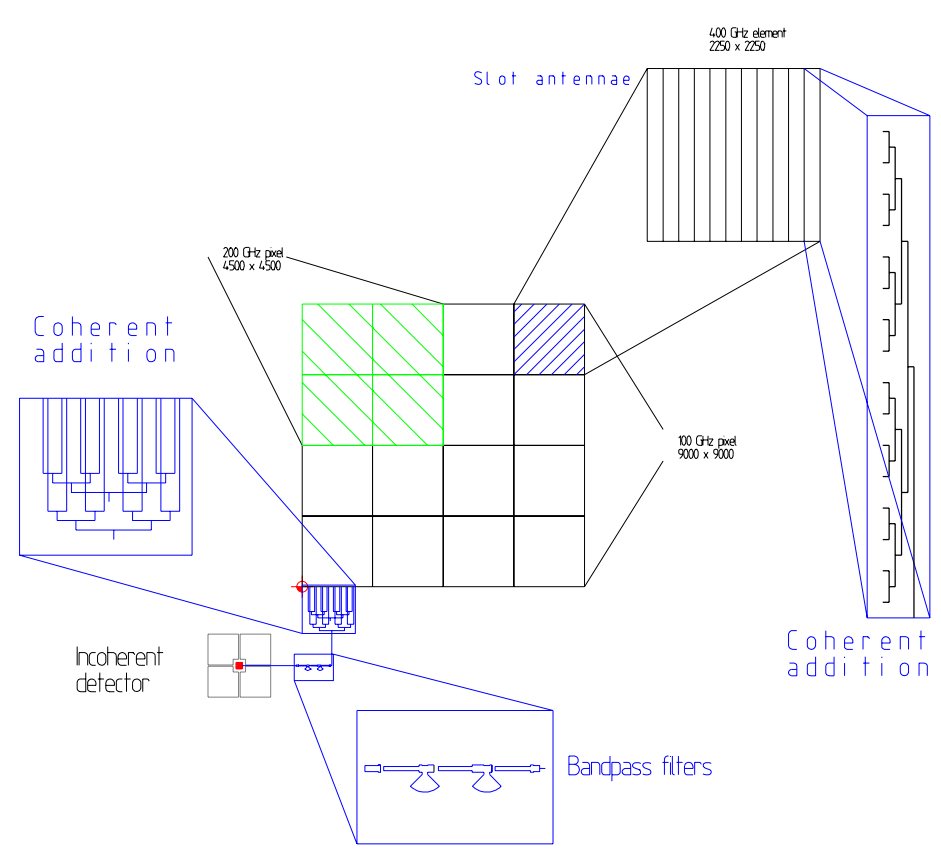

Figure 7. Multi-frequency array unit cell

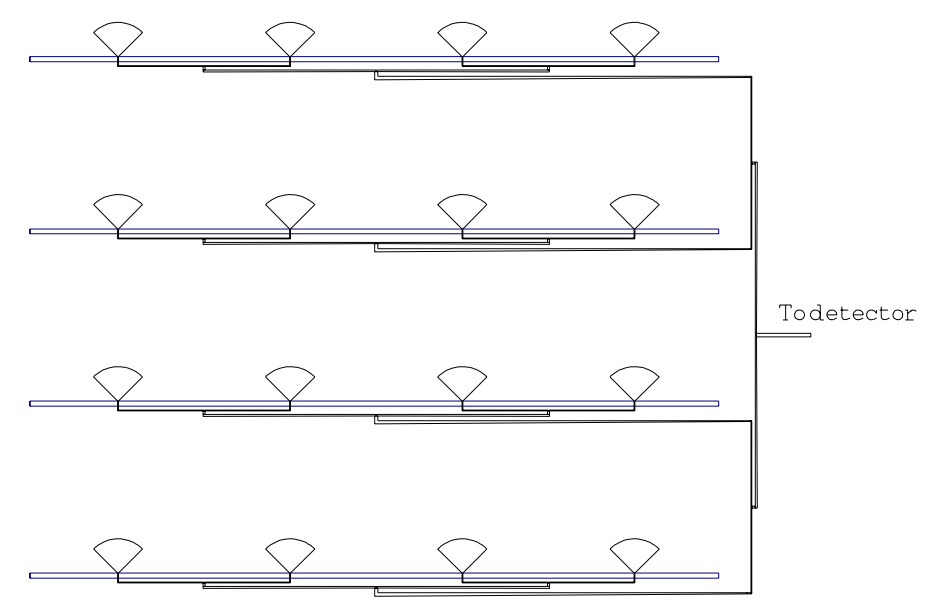

Figure 8. A small "baby" 4 by 4 single polarization array, shown for illustration purposes only. Dimensions are not to scale to avoid putting extremely fine details in a plot. 


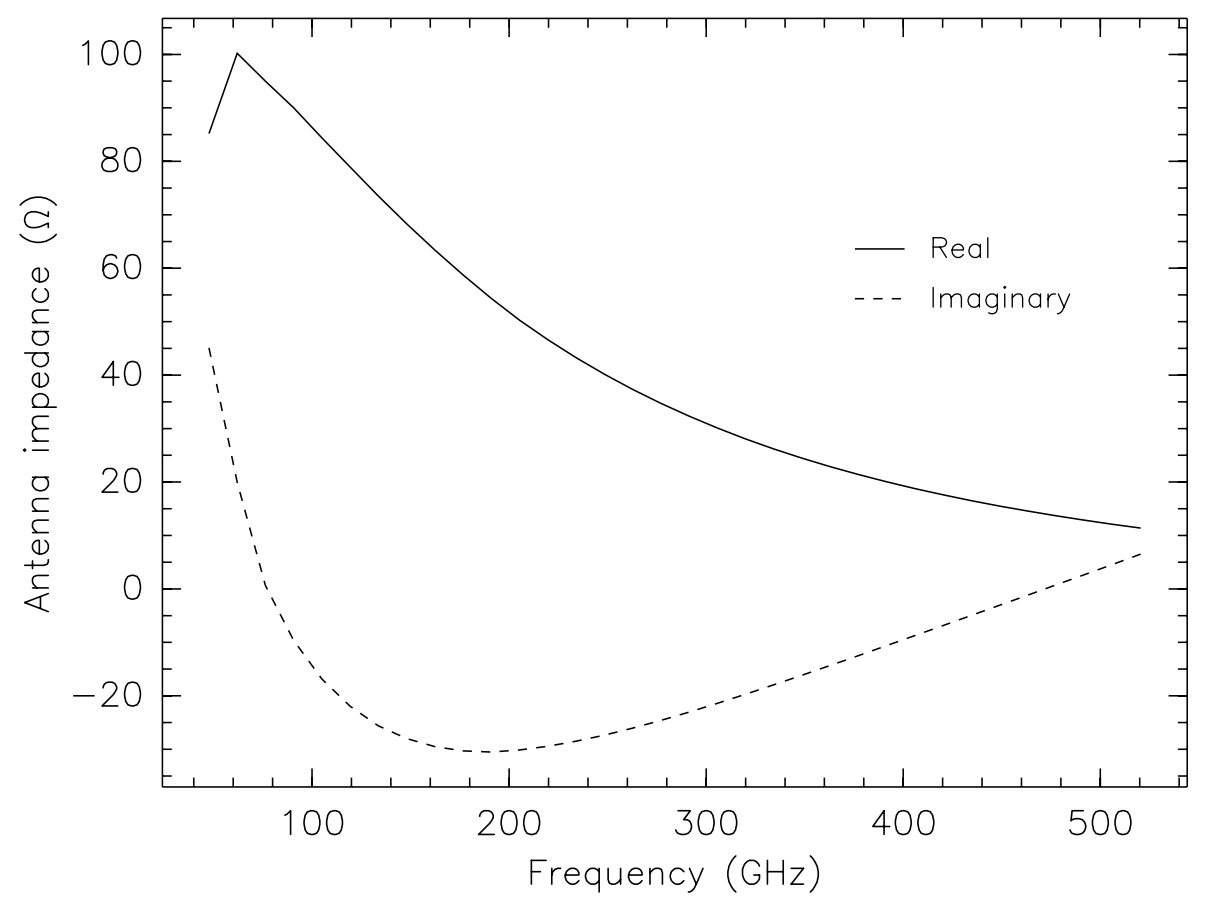

Figure 9. Impedance of antenna array shown on figure 8.

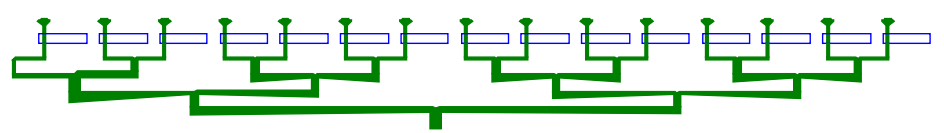

Figure 10. Feed network of one row of slots. Width of slots and lines is not to scale. At every split admittance $Y$ of every branch is proportional to number of slots it is feeding, assuring equal power is delivered to every slot. 

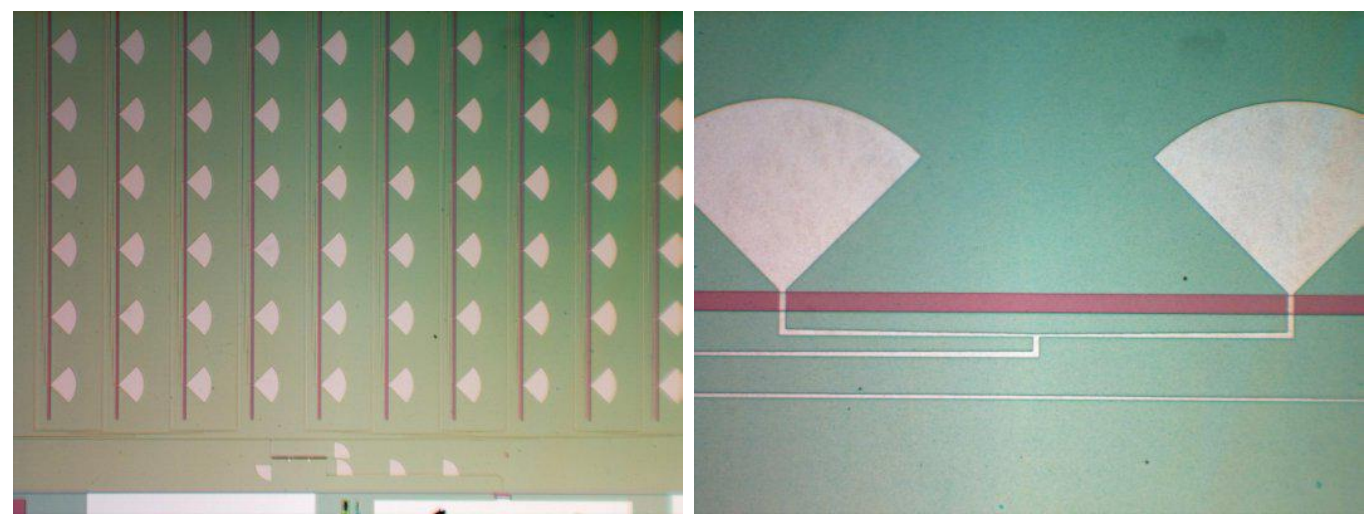

Figure 11. Antenna prototype manufactured at JPL

refraction coefficient can be used. A graded refraction index structure operating over a wide band can be realized with a stack of micromachined silicon wafers. For the dual polarization array, a tuned stack of quartz and teflon can be used.

\section{ANTENNA PROTOTYPE TESTING}

To test an antenna, we built a prototype with sixteen 20 micron wide slots. Each slot was tapped by 16 taps, all 256 taps combined into single microstrip line terminated by SIS junction used as detector. The test chip was cowered by one mm thick quartz wafer serving as simple antireflection coating.

The assembly was housed in a liquid helium dewar with a 2 inch window, approximately 1 inch behind the window. The vacuum window was made from a 40 micron mylar, with 25 micron Zytex plastic window at liquid nitrogen temperature for infrared blocking. stage -1 milliinch Zytex plastic. Using thin plastics helps to reduce standing waves.

The test dewar was placed on a machine shop rotating stage, and illuminated with a $126 \mathrm{GHz}$ Gunn diode modulated at $30 \mathrm{MHz}$. The signal was read out by Stanford Research SRS-844 lockin amplifier and recorded at one degree step.

The predicted and measured shapes are reasonable close, as can be seen in Figure 12. The dips in the measured shape are due to reflections inside the dewar.

\section{ACKNOWLEDGEMENTS}

This research was supported by NASA grant NAG5-10317. Alexey Goldin is supported by NRC Associateship stipendary award.

\section{REFERENCES}

1. H. J. Blinchikoff and A. I. Zverev, Filtering in the time and frequency domains, Wiley, New York, 1976.

2. J. Ward, F. Rice, G. Chattopadhay, and J. Zmuidzinas, "Supermix: a flexible software library for high frequency circuit simulation, including sis mixers and superconducting elements," in Proceedings, Tenth International Symposium on Space Terahertz Technology, 1999. http://www.submm.caltech.edu/supermix.

3. J. Zmuidzinas, H. G. Leduc, and J. A. Stern, "Slot antenna sis mixers for submillimeter wavelengths," in Michigan Univ., The Third International Symposium on Space Terahertz Technology: Symposium Proceedings p 234 (SEE N93-27726 10-31), pp. 234+, 1992.

4. G. V. Eleftheriades and G. M. Rebeiz, "Self and mutual admittance of slot antennas on a dielectric halfspace.," IRMMW , July 1993. 


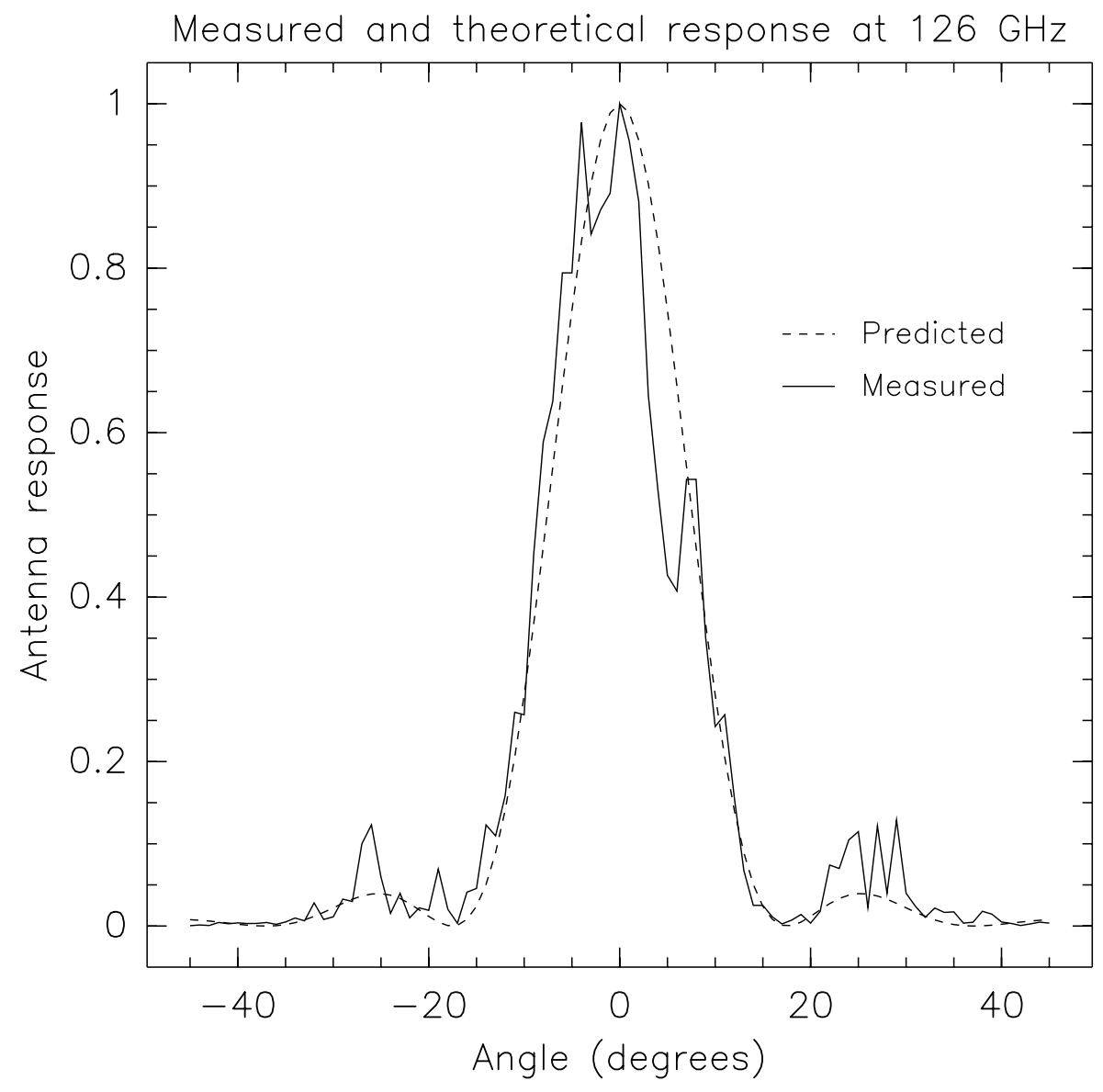

Figure 12. Measured and predicted beamshape

5. L. Vayonakis, A., "The millimeter-wave properties of superconducting microstrip lines," in Far-Infrared, Sub-MM and MM Detector Workshop, Monterey, CA, Apr. 2002. 